\title{
Liderazgo actual: una percepción de estudiantes de educación superior
}

\section{Current leadership: a perception of higher education students}

\author{
VELASCO-CEPEDA, Raquel Ivonne $\dagger^{*}$, CLARK-MENDÍVIL, Yesenia y ESPARZA-GARCÍA, Irma \\ Guadalupe
}

Instituto Tecnológico de Sonora, Departamento de Ciencias Administrativas. Calle 5 de Febrero 818, Centro, Urb. No. 1, 85000 Cd Obregón, Son.

ID $1^{\text {er }}$ Autor: Raquel Ivonne, Velasco-Cepeda / ORC ID: 0000-0003-3920-3537, Researcher IDThomson: S-7708-2018, CVU CONACYT ID: 673322

ID $1^{\text {er }}$ Coautor: Yesenia, Clark-Mendívil / ORC ID: 0000-0003-2032-7433, Researcher ID Thomson: S-7878-2018, CVU CONACYT ID: 290473

ID $2^{\text {do }}$ Coautor: Irma Guadalupe, Esparza-García / ORC ID: 0000-0002-3715-0761, Researcher Clarivate Analytics ID: 3090315, CVU CONACYT ID: 287445

DOI: 10.35429/JET.2019.7.3.1.12

Recibido: 03 de Enero, 2019; Aceptado 27 de Marzo, 2019

\section{Resumen}

Objetivo del estudio identificar la percepción de los estudiantes de licenciatura en administración sobre el liderazgo ante los retos actuales, los valores y cualidades que requiere el líder de hoy, así como los ámbitos en donde se encuentran los dilemas éticos, para el desarrollo de competencias que incidan en la solución de problemas de su profesión. Investigación de tipo no experimental, transeccional, descriptiva, enfoque cuantitativo. Una sección del instrumento tuvo una escala de 5 categorías de respuesta, la segunda 3: muy importante, regularmente importante y poco importante; tercera con tres: muchos, algunos y ninguno. Los resultados ante las problemáticas actuales obtuvieron las medias más altas en: el liderazgo actual necesita de la conducta ética y moral con 4.41, la sociedad no ha tomado conciencia de sus grandes problemas con 4.23, las personas están decepcionadas por falta de voluntad de las autoridades con 4.21. Sobre la percepción de los valores y cualidades del líder: ser honesto 1.13, tener claro los objetivos que quiere alcanzar 1.14 y capacidad para enfrentar retos 1.16. Los ámbitos en donde se encuentran los problemas éticos: 1.40 en lo educativo, económico 1.48, y 1.51 en liderazgo. El estudio contribuye para futuras investigaciones y al estado del arte.

Liderazgo, Líder, Ética

\begin{abstract}
Objective of the study to identify students' perception of the degree in administration about leadership in the face of current challenges, the values and qualities required by today's leader, as well as the areas where ethical dilemmas are, for the development of competences that affect the solution of problems of their profession. Nonexperimental, transectional, descriptive, quantitative approach research. One section of the instrument had a scale of 5 response categories, the second with 3: very important, regularly important and unimportant; third with three: many, some and none. The results before the current problems obtained the highest means in: the current leadership needs ethical and moral conduct with 4.41, society has not become aware of its great problems with 4.23; people are disappointed by the unwillingness of the authorities with 4.21. On the perception of the values and qualities of the leader: be honest 1.13 , be clear about the objectives you want to achieve 1.14 and ability to face challenges 1.16. The areas where ethical problems are found: 1.40 in education, economic 1.48 , and 1.51 in leadership. The study contributes to future research and the state of the art.
\end{abstract}

Leadership, Leader, Ethics

Citación: VELASCO-CEPEDA, Raquel Ivonne, CLARK-MENDÍVIL, Yesenia y ESPARZA-GARCÍA, Irma Guadalupe. Liderazgo actual: una percepción de estudiantes de educación superior. Revista Teoría Educativa. 2019. 3-7: 1-12.

\footnotetext{
* Correspondencia del Autor (correo electrónico: raquel.velasco@itson.edu.mx)

$\dagger$ Investigador contribuyendo como primer autor.
} 


\section{Introducción}

Las organizaciones se encuentran inmersas en un ambiente competitivo y que cambia de forma vertiginosa, en un entorno más globalizado, con recursos humanos más cualificados y con una mayor capacidad de comunicación e información debido a los avances tecnológicos; exigiendo que el líder no sólo debe ajustarse a estas condiciones, sino que también obtener de ellas el mayor provecho, para ello se debe de contar con un liderazgo efectivo y flexible (Contreras, 2008). Asimismo, Terrazas (2015) explica que los adelantos de la humanidad son sorprendentes y han alcanzado altos niveles de sofisticación, sin embargo, el avance tecnológico no ha logrado resolver los problemas complejos a los que se enfrenta el ser humano, como lo son: económicos, políticos y morales, éticos, de salud, entre otros. Hay un crecimiento exponencial en la tecnología, mientras que los valores y principios han tenido un decrecimiento exponencial. Lo anterior se observa en las noticias sobre atentados políticos, secuestros, catástrofes, corrupción, ocasionando descontentos en las personas. El mismo autor hace referencia que el ser humano en la sociedad del conocimiento se ha convertido en un personaje que exige derechos, pero no desea asumir responsabilidades.

Por otra parte, los líderes y presidentes de las naciones, empiezan a mostrar características de dictadores, deseando convertirse en hombres de absoluto poder; creyendo que con su dominio y estrategias solucionarán todos los problemas. En la actualidad, el entorno empresarial se ha caracterizado reiteradamente por comportamientos de carácter inmoral, por lo que la sociedad está demandando cada vez más un comportamiento ético (Ruiz-Palomino, RuizAmaya y Martínez-Cañas, 2012). En ese mismo orden de ideas, Perrone, Engelman, Reppold y Reinaldo (2013), hacen referencia a que las empresas con el fin de equilibrar las necesidades organizacionales y las sociales, y de esa manera resolver los dilemas éticos, han propuesto códigos de conducta, valores, gerencias con responsabilidad social, entre otros; de tal manera que el dilema ético se convierte para la organización en una imagen positiva. Existen diversos factores que influyen en el comportamiento ético y no ético del empleado en las empresas, y respecto a ello Ruíz-Palomino y Ruiz-Amaya (2011) mencionan que son de dos tipos: el contexto organizacional y el individual.
En el primero de los casos se tiene como referente al liderazgo y a otros miembros de la organización, ya que el comportamiento se aprende al interactuar con otras personas. También la organización elabora documentos formales para regular el comportamiento, tal como lo mencionó Perrone et al. (2013). El segundo de los casos está relacionado con las características del individuo, esto es, el control que tenga de sí mismo sobre los acontecimientos de su vida, el sistema de valores, la religiosidad; los cuales son determinantes de su comportamiento ético o no ético.

Las universidades juegan un papel relevante en la formación integral de sus estudiantes, tal es el caso del Instituto Tecnológico de Sonora (ITSON) cuya misión como universidad pública y autónoma, es su compromiso con la formación de profesionistas íntegros, competentes y emprendedores, la generación y aplicación del conocimiento y la extensión de la ciencia, la cultura y el deporte, para contribuir al desarrollo sostenible de la sociedad. Lo anterior da la pauta para formar individuos con virtudes morales, éticas y con capacidad de enfrentar los retos del entorno. Las organizaciones demandan líderes éticos y comprometidos con su desempeño y el de la empresa.

Los alumnos de la Licenciatura en Administración del ITSON durante su carrera abordan temas relacionados con el liderazgo, ya que como futuros profesionistas se espera que sean líderes de su propia empresa o en su caso ocupen un puesto con personal a su cargo. Y como menciona González y González (2008), un buen liderazgo requiere crear condiciones que aseguren una constante participación, y no solo estar como espectador, sino que debe ser un sujeto activo del proceso, y esto solamente se puede lograr en la manera en la que el líder maneje un liderazgo eficiente en la dinámica de su desempeño laboral.

En un estudio realizado por Omiaziki (2007) cuyo objetivo fue el identificar los grandes problemas contemporáneos, así como los valores y las cualidades más importantes que se requieren para un liderazgo ético, desde la perspectiva de los estudiantes de universidades tanto públicas como privadas. La muestra estuvo integrada por 100 alumnos. 
El autor utilizó un cuestionario integrado por cuatro secciones: (a) datos demográficos, (b) identificación de los elementos asociados a los grandes problemas del momento, (c) opiniones sobre los valores y cualidades que requiere el liderazgo de hoy, y (d) percepción de los estudiantes sobre los ámbitos en donde se encuentran los grandes problemas. Se utilizó una escala de Likert; en la sección (b), se presentaron cinco opciones de respuesta (totalmente en desacuerdo, en desacuerdo, ni en desacuerdo ni de acuerdo, de acuerdo y totalmente de acuerdo); en la sección (c ), se mostraron tres opciones (muy importante, regularmente importante, y poco importante). La última sección fueron tres opciones (muchos, algunos y ninguno).

Los resultados obtenidos respecto a los principales problemas del momento se encontró la media más alta en el reactivo: cada quien busca su beneficio propio, con 4.34; seguido del ítem: la sociedad no ha tomado conciencia de sus grandes problemas con una media de 4.33. Otro hallazgo encontrado con una media de 4.32 es el relacionado a que las personas están decepcionadas por la falta de voluntad de sus autoridades. Continuando con el mismo orden, se tiene que el liderazgo actual necesita mucho de la conducta ética y moral, el cual obtuvo una media de 4.28, seguida de los líderes manipulan a la gente sin educación con 4.14. Por otra parte, se obtuvo una media de 4.10 en donde los informantes manifestaron que no les gusta que alguien le imponga sus maneras de pensar, ni de vivir. Entre otras opiniones, se encontró que cada vez cuesta más concebir a las religiones como portadoras de valores con una media de 3.5. Los estudiantes consideran que hay cada vez más gente que no cumple con sus responsabilidades, y también que se han perdido sus valores con unas medias de 3.93 y 3.91 respectivamente.

Continuando con los hallazgos encontrados, se tiene que los estudiantes piensan que es cada vez más frecuente ver a la gente que considera la corrupción como algo normal con una media de 3.69 , seguida de cada persona tiene su verdad, con una media de 3.67. Además, los informantes manifestaron que: la gente habla más de sus obligaciones que de sus derechos con una media de 3.54; y en contraparte se encontró que la gente habla más de sus derechos que de sus obligaciones con un 3.41.
Respecto a las cualidades y valores que requiere el líder se encontraron los siguientes resultados con sus respectivas medias: ser audaz (1.40), buscar y decir la verdad (1.24), fomentar el diálogo (1.16) y la responsabilidad moral (1.03). Cabe hacer mención que los valores de la escala fluctúan de la siguiente manera: muy importante (1), regularmente importante (2), y poco importante (3). Por lo anterior, los resultados mostraron que la responsabilidad es la más importante, seguido por el diálogo, la búsqueda de la verdad, y por último el valor de la audacia.

En la sección relacionada con el ámbito en donde se encuentran los problemas y dilemas éticos, se tiene que principalmente están en lo económico con un promedio de 1.10, seguido de lo político con 1.16, en lo educativo con 1.24 , la familia 1.39, liderazgo con 1.50 , relaciones personales 1.73 , y por último en los religioso con 1.80.

\section{Problema}

Los escándalos éticos se han vuelto comunes en la actualidad en empresas de diferentes giros, en los deportes, la política, en la industria del entretenimiento. Estos escándalos han sacudido la confianza de los grupos de interés y cuestionado la integridad moral de nuestra sociedad. Aunado a lo anterior, el liderazgo ético está basado en la premisa de que los líderes sirven como modelos a seguir, por lo que todas sus acciones están bajo una lupa, y como directivos deben de establecer el tono moral de la empresa al ser honestos y directos; así como, asumir la responsabilidad por cualquier deficiencia identificada (Griffin, 2011).

Los futuros profesionistas egresados de la licenciatura en administración deberán practicar un liderazgo ético y para ello contar con los valores y cualidades para hacer frente a los retos del entorno. Así mismo, deben ser reflexivos y personas con criterio para evaluar las problemáticas actuales y realizar acciones encaminadas en beneficio de la sociedad.

Por lo anterior surge el siguiente planteamiento: 
¿Cuál es la percepción del estudiante de la licenciatura en administración de una institución de educación superior sobre el liderazgo ante los retos actuales, los valores y cualidades que requiere el líder, así como los ámbitos en donde se encuentran los dilemas éticos?

\section{Objetivo}

Identificar la percepción de los estudiantes de la licenciatura en administración sobre el liderazgo ante los retos actuales, los valores y cualidades que requiere el líder de hoy, así como los ámbitos en donde se encuentran los dilemas éticos, para el desarrollo de competencias que incidan en la solución de problemas de su profesión.

\section{Justificación}

El estudio del liderazgo ante los retos actuales adquiere relevancia ante la problemática planteada en párrafos anteriores y como menciona Terrazas (2015), el liderazgo es determinante en el desempeño de las sociedades y puede ser concebido en diferentes ámbitos de las organizaciones y grupos humanos; se trata de comprender la necesidad que todos tienen de una guía para poder caminar en el quehacer de la vida. Por otra parte, este tipo de estudio ayuda a identificar parámetros hacia donde se puede inclinar la toma de decisiones éticas o no éticas, así como esclarecer el perfil del líder.

La investigación permitió profundizar en los conceptos relacionados al liderazgo, al líder, la ética y como menciona Méndez (1995), el marco teórico lo constituye la presentación de postulados según diversos autores e investigadores que proporcionan una visión completa para la formulación del conocimiento científico, y a su vez establece las relaciones que se dan entre los elementos teóricos.

Los resultados obtenidos de la investigación permitieron obtener información directamente del estudiante sobre su percepción en relación a la temática del liderazgo ante los retos actuales y si en un momento dado cuenta con los conocimientos, habilidades y destrezas como futuro profesionista para enfrentarlos, y sobre todo a actuar de manera proactiva y ética.

\section{Marco teórico}

Para dar soporte a esta investigación, a continuación, se presenta el marco teórico que sirvió como referencia del estudio: liderazgo, líder, ética y dilemas éticos.

\section{Liderazgo}

El liderazgo ha sido definido por diversos autores, Lussier y Achua (2011) lo definen como: "el proceso de influencia entre líderes y seguidores para lograr los objetivos organizacionales por medio del cambio" (pag.6). Por otra parte, Franklin y Krieger (2011) hacen referencia al liderazgo como "la capacidad de influir en las actitudes de una persona, grupo o equipo que forma parte de una empresa y está orientado a alcanzar objetivos y metas en cierta situación" (pag.294). Otra aportación sobre el liderazgo es la realizada por Prieto y Favelo (2009), quienes dicen que este es ejercido en cualquier ámbito de la vida, y está estrechamente relacionado con la educación que puede haber recibido el líder, en su familia, la escuela, su universidad en relación al fomento de la ética y los valores del respeto, la confianza, la responsabilidad, la comprensión, la solidaridad, entre otros.

\section{Líder}

El líder es la persona que es capaz de ejercer influencia en otros, de tal manera que puede dirigirlos y guiarlos hacia el logro de metas organizacionales (Gómez, 2008). Etling, 2005 (citado por Vázquez, Bernal \& Liesa, 2014) define al líder como una persona que influye sobre los demás; y éste puede ser inteligente, persuasivo, emprendedor y con poder de convencimiento. Por otra parte, Castro, Clemenza \&Araujo (2012) hacen mención que la tarea principal de un líder es el de construir organizaciones y sistemas que creen la armonía entre las necesidades individuales y de la organización.

Un líder debe desarrollar ciertas cualidades y conocimientos para poder desempeñar bien su función de dirección en las organizaciones, para ello Münch (2010) describe el siguiente perfil: (a) conocimientos tecnológicos, (b) conocimientos administrativos y (c) competencias personales. 
Respecto a éstas últimas, las cualidades que más sobresalen son: visión, autocontrol, seguridad en sí mismo, creatividad e iniciativa, sentido común, actitud positiva, así como sinceridad, justicia y lealtad.

\section{Ética}

En este apartado se analizarán dos conceptos relacionados, lo que es la ética y el comportamiento ético. La ética es definida por Williams (2013), como "el conjunto de principios o valores morales que definen lo que es correcto e incorrecto para una persona o grupo" (pag.65). En el mismo sentido, Hellriegel y Slocum (2009) la definen como "los valores y los principios que distinguen el bien y el mal" (pag.16). Estos principios que mencionan ambos autores es lo que va a dar origen al comportamiento ético; el cual es definido por Wiliams (2013) como aquel comportamiento que se ajusta a los principios que son aceptados por la sociedad sobre lo que es correcto e incorrecto.

\section{Dilema ético}

Un dilema ético es una condición que se presenta cuando dos preceptos morales o las obligaciones de semejante obligatoriedad ética se encuentran en conflicto, de tal forma que cualquier solución posible al dilema es moralmente intolerable (Ruíz-Cano, et al., 2015). Se puede observar, tal como lo menciona Ferrer y Clemenza (2006) que cada grupo, organización y/o comunidad posee una moral cruzada por rasgos valorativos, que en ocasiones son compartidos $y$ en ciertas circunstancias conflictivas, que se presentan en las conductas, las actitudes, convicciones y sus creencias sobre ellas, lo que pone en evidencia la ética.

La ética indiscutiblemente está relacionada con los valores, y Álvarez del Blanco (2008) menciona que éstos continuamente entran en conflicto por que las personas difieren de lo que constituyen sus propios valores. Llama la atención un término que utiliza, el relacionado con la prueba crítica de los valores, y menciona que es el estrés; y dice que es más fácil comprometerse a ellos en situaciones de baja presión y la prueba llega ante situaciones en la que la presión es mayor.
Nelson y Quick (2013) mencionan que las organizaciones enfrentan una variedad de dilemas éticos y morales, entre los que se encuentran los derechos de los empleados (estos se ven afectados por pruebas de antidopaje, libertad de expresión, discapacidad, downsizing y despidos, entre otros), acoso sexual, justicia organizacional (distributiva y procesal). Lo anterior ha propiciado la necesidad de establecer códigos de conducta y crear conciencia de su responsabilidad social.

\section{Metodología}

La investigación es de tipo no experimental, transeccional, descriptiva con enfoque cuantitativo.

\section{Sujetos de estudio}

Los sujetos de estudio fueron un total de 176 estudiantes de la licenciatura en administración del Campus Centro de Ciudad Obregón, inscritos en el semestre de enero-mayo de 2017.

\section{Instrumentos}

El instrumento que se tomó como referencia fue el utilizado por Omiaziki (2007). El cuestionario estuvo conformado por cuatro secciones. Una de ellas fue la de datos generales del informante en donde se preguntaba la edad, el género y el semestre que cursa. Otra sección estuvo relacionada con las problemáticas actuales con 13 ítems, con categorías de respuesta en escala de Likert: (1) totalmente en desacuerdo, (2) en desacuerdo, (3) ni de acuerdo, ni en desacuerdo, (4) de acuerdo, y (5) totalmente de acuerdo. La sección relacionada con los valores y cualidades que requiere el liderazgo de hoy, estuvo conformada por 11 enunciados. La escala de respuesta que se utilizó fue la siguiente: (1) muy importante, (2) regularmente importante, y (3) poco importante. La última sección del cuestionario fue la referente a los ámbitos en donde se encuentran los problemas o dilemas éticos, con un total de 8 ítems y con las siguientes categorías de respuesta: (1) muchos, (2) algunos, y (3) ninguno.

En la tabla 1 se muestra las variables de estudio con sus respectivos ítems. 


\begin{tabular}{|c|c|}
\hline Variable & Ítems \\
\hline $\begin{array}{l}\text { Problemáticas } \\
\text { actuales }\end{array}$ & $\begin{array}{l}\text { 1. Cada quien busca su propio } \\
\text { beneficio. } \\
\text { 2. Pienso que nuestra sociedad } \\
\text { no ha tomada conciencia de } \\
\text { sus grandes problemas. } \\
\text { 3. Las personas están } \\
\text { decepcionadas por la falta de } \\
\text { voluntad de sus autoridades. } \\
\text { 4. El liderazgo actual necesita } \\
\text { mucho de la conducta ética y } \\
\text { moral. } \\
\text { 5. Los líderes manipulan a la } \\
\text { gente sin educación. } \\
\text { 6. No me gusta que alguien me } \\
\text { impongan sus maneras de } \\
\text { pensar, ni de vivir. } \\
\text { 7. Hay cada vez más gente que } \\
\text { no cumple con sus } \\
\text { responsabilidades. } \\
\text { 8. Siento que se han perdido los } \\
\text { valores. } \\
\text { 9. Es cada vez más frecuente ver } \\
\text { a la gente que considera a la } \\
\text { corrupción como algo normal. } \\
\text { 10. Cada persona tiene su verdad. } \\
\text { 11. Cada vez cuesta más concebir } \\
\text { a las religiones como } \\
\text { portadoras de valores. } \\
\text { 12. Muchos hablan más de sus } \\
\text { obligaciones que de sus } \\
\text { derechos. } \\
\text { 13. Muchos hablan más de sus } \\
\text { derechos que de sus } \\
\text { obligaciones. }\end{array}$ \\
\hline $\begin{array}{l}\text { Valores } \\
\text { cualidades } \\
\text { requeridos por el } \\
\text { liderazgo }\end{array}$ & $\begin{array}{l}\text { 14. Tener capacidad para enfrentar } \\
\text { los retos. } \\
\text { 15. Ser coherente. } \\
\text { 16. Ser honesto. } \\
\text { 17. Ser responsable hacia los } \\
\text { demás. } \\
\text { 18. Tener carácter. } \\
\text { 19. Ser perseverante } \\
\text { 20. Tener claro los objetivos que } \\
\text { quieren alcanzar. } \\
\text { 21. Buscar y decir siempre la } \\
\text { verdad. } \\
\text { 22. Ser responsable. } \\
\text { 23. Fomentar el diálogo. } \\
\text { 24. Ser audaz. }\end{array}$ \\
\hline $\begin{array}{lr}\text { Ámbitos } & \text { en } \\
\text { donde } & \text { se } \\
\text { encuentran } & \text { los } \\
\text { problemas } & \text { o } \\
\text { dilemas éticos. } & \end{array}$ & $\begin{array}{l}\text { 25. Relaciones personales. } \\
\text { 26. Liderazgo. } \\
\text { 27. Religioso. } \\
\text { 28. Familiar. } \\
\text { 29. Cultural. } \\
\text { 30. Educativo. } \\
\text { 31. Político. } \\
\text { 32. Económico. }\end{array}$ \\
\hline
\end{tabular}

Tabla 1 Variables de estudio con sus respectivos ítems Fuente: elaboración a partir del instrumento

\section{Procedimiento}

Para la realización de la presente investigación, se consideraron como referencia los pasos realizados por Lamb, Hair y McDaniel (2011), y el de McDaniel y Gates (2011); realizándose los ajustes pertinentes al estudio, los cuales se describen a continuación:

(a) Definir el problema así como los requerimientos de información. Se definió el planteamiento del problema, y se realizó una búsqueda de información relacionada a la temática del liderazgo y dilemas éticos, los cuales sirvieron de referencia para la contextualización

(b) Especificar la fuente de datos primarios. Se decidió recolectar información directamente de los estudiantes de la licenciatura en administración con el fin de conocer su percepción sobre el liderazgo y los dilemas éticos.

(c) Definir el tamaño de la muestra. El tipo de muestreo utilizado fue no probabilístico y por conveniencia del investigador. También se definió que se seleccionarían los alumnos de cursos presenciales y no los virtuales.

(d) Revisar instrumentos sobre la temática. Se definió el utilizar el de Omiaziki (2007), utilizando las mismas tres variables: problemas de nuestros tiempos, valores y cualidades que requiere el liderazgo de hoy, y ámbitos en donde se encuentran los problemas y dilemas éticos.

(e) Aplicar los cuestionarios. Para la aplicación de los cuestionarios se solicitó a la Jefatura del Departamento el catálogo de grupos programados durante el semestre de eneromayo de 2017. Se visitaron los grupos pidiendo autorización a los profesores y la participación de los alumnos.

(f) Revisar los cuestionarios aplicados. La revisión se realizó para cotejar si la información proporcionada era completa y coherente.

(g) Capturar y procesar la información en el paquete estadístico SPSS versión 21. Previamente a ello se elaboró un libro de códigos para la facilitación de la captura. 
(h) Realizar la prueba de validez del instrumento. En la tabla 2 se presenta la prueba de validez del instrumento, que determina la consistencia interna de los ítems y cuyos coeficientes son superiores a .7 y de acuerdo a George y Mallery (2003) son valores aceptables.

\begin{tabular}{|l|c|c|}
\hline \multicolumn{1}{|c|}{ Variable } & \multicolumn{1}{|c|}{$\begin{array}{l}\text { Ítems } \\
\text { inicial }\end{array}$} & \multicolumn{1}{c|}{$\begin{array}{c}\text { Alfa de } \\
\text { Cronbach }\end{array}$} \\
\hline $\begin{array}{l}\text { La percepción sobre las } \\
\text { problemáticas actuales. }\end{array}$ & 13 & .733 \\
\hline $\begin{array}{l}\text { Valores y cualidades que } \\
\text { requiere el liderazgo de } \\
\text { hoy. }\end{array}$ & 11 & .776 \\
\hline
\end{tabular}

Tabla 2 Validez del instrumento

Fuente: elaboración a partir de los resultados

(i) Graficar y tabular los datos. Se realizaron gráficos y tablas para la interpretación de los mismos.

(j) Analizar e interpretar la información. Para el análisis e interpretación de la información se calcularon las medias de cada uno de los reactivos de las variables estudiadas.

(k) Identificar la percepción de los estudiantes respecto al liderazgo ante los retos actuales, los valores y cualidades que requiere el líder de hoy y los ámbitos en donde se encuentran los dilemas éticos.

\section{Resultados}

A continuación, se presentan los resultados de la investigación:

\section{(a) Caracterización de los informantes}

En la tabla 3 se presentan las características de los informantes que participaron en el estudio.

\begin{tabular}{|l|r|r|}
\hline \multicolumn{1}{|c|}{ Características } & \multicolumn{1}{c|}{ N } & \\
\hline Género & 103 & 58.5 \\
\hline Femenino & 73 & 41.5 \\
\hline Masculino & & \\
\hline Edad & 128 & 73 \\
\hline $19-22$ & 34 & 19 \\
\hline $23-25$ & 14 & 8 \\
\hline Más de 25 en & 176 & 100 \\
\hline Programa Educativo & & \\
\hline $\begin{array}{l}\text { Licenciado } \\
\text { Administración }\end{array}$ & 10 & 5.7 \\
\hline Semestre & 78 & 44.3 \\
\hline Quinto & & \\
\hline Sexto & & \\
\hline
\end{tabular}

ISSN 2523-2509

ECORFAN® Todos los derechos reservados

\begin{tabular}{|l|l|l|}
\hline Séptimo & 29 & 16.5 \\
\hline Octavo & 59 & 33.5 \\
\hline
\end{tabular}

Tabla 3 Caracterización de los informantes Fuente: elaboración a partir de los resultados

Como se puede observar la edad que predominó fue en un rango de 19 a 22 años. Asimismo, el género femenino tuvo una participación mayor con un $58.5 \%$. La mayoría de los integrantes de la muestra estuvo conformado por alumnos del sexto semestre con un $44.3 \%$ y seguido del octavo con un $33.5 \%$. Una razón que lo justifica es que el semestre enero-mayo corresponde a los pares (2do, 4to, 6to y 8vo). Cabe aclarar que los semestres del 5to al 8vo de la carrera se ofrecen en el Campus Centro Obregón, y los primeros en el Campus Náinari Obregón.

(b) Percepción de los estudiantes sobre las problemáticas actuales

En la tabla 4 se presentan los estadísticos descriptivos sobre la percepción de las problemáticas actuales.

\begin{tabular}{|l|c|c|}
\hline \multicolumn{1}{|c|}{ Criterio de valoración } & Media & $\begin{array}{c}\text { Desv. } \\
\text { típ }\end{array}$ \\
\hline $\begin{array}{l}\text { 1. Cada quien busca su propio } \\
\text { beneficio. }\end{array}$ & 3.82 & 1.145 \\
\hline $\begin{array}{l}\text { 2. Pienso que nuestra sociedad no ha } \\
\text { tomado conciencia de sus grandes } \\
\text { problemas. }\end{array}$ & 4.23 & .947 \\
\hline $\begin{array}{l}\text { 3. Las personas están decepcionadas } \\
\text { por la falta de voluntad de sus } \\
\text { autoridades. }\end{array}$ & 4.21 & .966 \\
\hline $\begin{array}{l}\text { 4. El liderazgo actual necesita } \\
\text { mucho de la conducta ética y moral. }\end{array}$ & 4.41 & .870 \\
\hline $\begin{array}{l}\text { 5. Los líderes manipulan a la gente } \\
\text { sin educación. }\end{array}$ & 3.90 & 1.117 \\
\hline $\begin{array}{l}\text { 6. No me gusta que alguien me } \\
\text { imponga sus maneras de pensar, ni } \\
\text { de vivir. }\end{array}$ & 4.14 & .946 \\
\hline $\begin{array}{l}\text { 7. Hay cada vez más gente que no } \\
\text { cumple con sus responsabilidades. }\end{array}$ & 4.18 & .820 \\
\hline $\begin{array}{l}\text { 8. Siento que se han perdido los } \\
\text { valores. }\end{array}$ & 4.06 & .963 \\
\hline $\begin{array}{l}\text { 9. Es cada vez más frecuente ver a la } \\
\text { gente que considera a la corrupción } \\
\text { como algo normal. }\end{array}$ & 4.11 & .827 \\
\hline 10. Cada persona tiene su verdad. & 3.90 & .992 \\
\hline $\begin{array}{l}\text { 11. Cada vez cuesta más concebir a } \\
\text { las religiones como portadoras de } \\
\text { valores. }\end{array}$ & 3.99 & .923 \\
\hline $\begin{array}{l}\text { 12. Muchos hablan más de sus } \\
\text { obligaciones que de sus derechos. }\end{array}$ & 3.25 & 1.281 \\
\hline $\begin{array}{l}\text { 13. Muchos hablan más de sus } \\
\text { derechos que de sus obligaciones. }\end{array}$ & 3.77 & 1.035 \\
\hline $\begin{array}{l}\text { Nota: N válido (según lista) 176 } \\
\text { Nota: mínimo 1, máximo 5 }\end{array}$ & \\
\hline
\end{tabular}

Tabla 4 Estadísticos descriptivos de la percepción sobre las problemáticas actuales

VELASCO-CEPEDA, Raquel Ivonne, CLARK-MENDÍVIL, Yesenia y ESPARZA-GARCÍA, Irma Guadalupe. Liderazgo actual: una percepción de estudiantes de educación superior. Revista Teoría Educativa. 2019 
Los resultados anteriores muestran que la media más alta la obtuvo el ítem: el liderazgo actual necesita mucho de la conducta ética y moral con 4.41, seguida de nuestra sociedad no ha tomado conciencia de sus grandes problemas cuya media es 4.23. La media de 4.21 la obtuvo el reactivo las personas están decepcionadas por la falta de voluntad de sus autoridades. Otra percepción que predominó con una media de 4.18 , fue la de cada vez hay más gente que no cumple con sus responsabilidades. La media más baja la obtuvo el reactivo de muchos hablan más de sus obligaciones que de sus derechos con una media de 3.25 .

(c) Percepción de los estudiantes sobre los valores y cualidades que requiere el liderazgo de hoy.

En la tabla 5 se presentan los estadísticos descriptivos de la percepción sobre los valores y cualidades que requiere el liderazgo de hoy. En su interpretación se tiene que (1) es muy importante, (2) regularmente importante, y (3) poco importante.

\begin{tabular}{|l|c|c|}
\hline \multicolumn{1}{|c|}{ Criterio de valoración } & Media & $\begin{array}{c}\text { Desv. } \\
\text { típ. }\end{array}$ \\
\hline $\begin{array}{l}\text { 1. Tener capacidad para enfrentar } \\
\text { los retos. }\end{array}$ & 1.16 & .411 \\
\hline 2. Ser coherente. & 1.20 & .466 \\
\hline 3. Ser honesto. & 1.13 & .400 \\
\hline $\begin{array}{l}\text { 4. Ser responsable hacia los } \\
\text { demás. }\end{array}$ & 1.22 & .468 \\
\hline 5. Tener carácter. & 1.32 & .502 \\
\hline 6. Ser perseverante. & 1.22 & .500 \\
\hline $\begin{array}{l}\text { 7. Tener claros los objetivos que } \\
\text { quieren alcanzar. }\end{array}$ & 1.14 & .424 \\
\hline $\begin{array}{l}\text { 8. Buscar y decir siempre la } \\
\text { verdad. }\end{array}$ & 1.28 & .611 \\
\hline 9. Ser responsable. & 1.22 & .883 \\
\hline 10. Fomentar el diálogo. & 1.37 & .561 \\
\hline 11. Ser audaz. & 1.36 & .559 \\
\hline $\begin{array}{l}\text { Nota: N válido (según lista) 176 } \\
\text { Nota: mín 1, max 3 }\end{array}$ & \\
\hline \multicolumn{2}{|l}{} \\
\hline
\end{tabular}

Tabla 5 Estadísticos descriptivos sobre los valores y cualidades que requiere el liderazgo de hoy

Respecto a la interpretación de la tabla anterior será de manera ascendente, la media más baja es la de mayor importancia dada por los estudiantes. Siendo la honestidad con una media de 1.13, seguida de tener claros los objetivos a alcanzar 1.14, tener capacidad para enfrentar los retos con 1.16, ser coherente con 1.20. Ser responsable y responsable ante los demás obtuvo la misma media 1.22, y ser perseverante 1.22.
Sin embargo, a las que le dieron menor importancia fue fomentar el diálogo con una media 1.37 , ser audaz 1.36 y tener carácter con 1.32 .

(d) Percepción sobre los ámbitos en donde se encuentran los problemas y dilemas éticos.

La tabla 6 muestra los estadísticos descriptivos en donde se encuentran los problemas y dilemas éticos.

\begin{tabular}{|l|r|r|}
\hline \multicolumn{1}{|c|}{ Criterio de valoración } & Media & Desv. Típ \\
\hline 1. Relaciones personales. & 1.51 & .566 \\
\hline 2. Liderazgo. & 1.51 & .545 \\
\hline 3. Religioso. & 1.78 & .724 \\
\hline 4. Familiar. & 1.65 & .677 \\
\hline 5. Cultura. & 1.77 & .639 \\
\hline 6. Educativo. & 1.40 & .587 \\
\hline 7. Político. & 1.56 & .746 \\
\hline 8. Económico. & 1.48 & .605 \\
\hline $\begin{array}{l}\text { Nota: N válido (según lista) 176 } \\
\text { Nota: mín.1, max 3 }\end{array}$ \\
\hline \multicolumn{2}{|l}{} \\
\hline
\end{tabular}

Tabla 6 Estadísticos descriptivos en donde se encuentran los problemas o dilemas éticos

Al igual que la interpretación de la tabla anterior esta se hace de manera ascendente. Encontrándose que la mayoría de los integrantes de la muestra perciben que los problemas se encuentran en el ámbito educativo con una media de 1.40, seguido en los económico con 1.48. En relaciones personales y el liderazgo se obtuvo una media igual a 1.51 respectivamente, seguido del ámbito familiar con 1.65. En último término se encontró en lo religioso con 1.78.

(e) Comparativo de los resultados obtenidos en la investigación y los hallazgos de Omiaziki (2007)

La tabla 7 muestra los resultados de las medias de la presente investigación y la de Omiaziki (2007), relacionadas a las problemáticas actuales.

\begin{tabular}{|c|c|c|}
\hline Criterio de valoración & Media & $\begin{array}{c}\text { Media } \\
\text { Omiaziki }\end{array}$ \\
\hline $\begin{array}{c}\text { 1. Cada quien busca su propio } \\
\text { beneficio. }\end{array}$ & 3.82 & 4.34 \\
\hline $\begin{array}{c}\text { 2. Pienso que nuestra sociedad } \\
\text { no ha tomado conciencia de } \\
\text { sus grandes problemas. }\end{array}$ & 4.23 & 4.33 \\
\hline 3. $\quad \begin{array}{c}\text { Las personas están } \\
\text { decepcionadas por la falta de } \\
\text { voluntad de sus autoridades. }\end{array}$ & 4.21 & 4.32 \\
\hline $\begin{array}{c}\text { 4. El liderazgo actual necesita } \\
\text { mucho de la conducta ética y } \\
\text { moral. }\end{array}$ & 4.41 & 4.28 \\
\hline
\end{tabular}

VELASCO-CEPEDA, Raquel Ivonne, CLARK-MENDÍVIL, Yesenia y ESPARZA-GARCÍA, Irma Guadalupe. Liderazgo actual: una percepción de estudiantes de educación superior. Revista Teoría Educativa. 2019 


\begin{tabular}{|l|l|l|}
\hline $\begin{array}{l}\text { 5. Los líderes manipulan a la } \\
\text { gente sin educación. }\end{array}$ & 3.90 & 4.14 \\
\hline $\begin{array}{l}\text { 6. No me gusta que alguien me } \\
\text { imponga sus maneras de } \\
\text { pensar, ni de vivir. }\end{array}$ & 4.14 & 4.10 \\
\hline $\begin{array}{l}\text { 7. Hay cada vez más gente que } \\
\text { no cumple con sus } \\
\text { responsabilidades. }\end{array}$ & 4.18 & 3.93 \\
\hline $\begin{array}{l}\text { 8. Siento que se han perdido los } \\
\text { valores. }\end{array}$ & 4.06 & 3.91 \\
\hline $\begin{array}{l}\text { 9. Es cada vez más frecuente ver } \\
\text { a la gente que considera a la } \\
\text { corrupción como algo } \\
\text { normal. }\end{array}$ & 4.11 & 3.69 \\
\hline $\begin{array}{l}\text { 10. Cada persona tiene su } \\
\text { verdad. }\end{array}$ & 3.90 & 3.67 \\
\hline $\begin{array}{l}\text { 11. Cada vez cuesta más } \\
\text { concebir a las religiones } \\
\text { como portadoras de valores. }\end{array}$ & 3.99 & 3.56 \\
\hline $\begin{array}{l}\text { 12. Muchos hablan más de sus } \\
\text { obligaciones que de sus } \\
\text { derechos. }\end{array}$ & 3.25 & 3.54 \\
\hline $\begin{array}{l}\text { 13. Muchos hablan más de sus } \\
\text { derechos que de sus } \\
\text { obligaciones. }\end{array}$ & 3.77 & 3.41 \\
\hline \multicolumn{2}{|c|}{ Nota: mínimo 1, máximo 5 } \\
\hline
\end{tabular}

Tabla 7 Comparativo de medias sobre la percepción de problemáticas actuales entre ambos estudios

Como se puede observar en la tabla anterior, las medias más altas obtenidas en los hallazgos de Omiaziki (2007) se presentaron en: cada quien busca su propio beneficio (4.34), pienso que nuestra sociedad no ha tomado conciencia de sus grandes problemas (4.33), las personas están decepcionadas por la falta de voluntad de sus autoridades (4.32). En el caso del presente estudio las medias más altas fueron: el liderazgo actual necesita mucho de la conducta ética y moral (4.41), seguida de la sociedad no ha tomado conciencia de sus grandes problemas (4.23), y las personas están decepcionadas por la falta de voluntad de sus autoridades (4.21). En algunos casos las medias entre los dos estudios estuvieron más semejantes, y en otros mayores diferencias.

A continuación, en la tabla 8 se muestran los resultados encontrados entre ambos estudios respecto a los valores y cualidades que requiere el liderazgo de hoy.

\begin{tabular}{|c|c|c|}
\hline Criterio de valoración & Media & $\begin{array}{c}\text { Media } \\
\text { Omiaziki }\end{array}$ \\
\hline $\begin{array}{l}\text { 1. Tener capacidad para } \\
\text { enfrentar los retos. }\end{array}$ & 1.16 & 1.06 \\
\hline 2. Ser coherente. & 1.20 & 1.08 \\
\hline 3. Ser honesto. & 1.13 & 1.07 \\
\hline $\begin{array}{l}\text { 4. Ser responsable hacia los } \\
\text { demás. }\end{array}$ & 1.22 & 1.13 \\
\hline 5. Tener carácter. & 1.32 & 1.06 \\
\hline
\end{tabular}

ISSN 2523-2509

ECORFAN $®$ Todos los derechos reservados

\begin{tabular}{|l|c|c|}
\hline 6. Ser perseverante. & 1.22 & 1.1 \\
\hline $\begin{array}{l}\text { 7. Tener claros los objetivos } \\
\text { que quieren alcanzar. }\end{array}$ & 1.14 & 1.01 \\
\hline $\begin{array}{l}\text { 8. Buscar y decir siempre la } \\
\text { verdad. }\end{array}$ & 1.28 & 1.24 \\
\hline 9. Ser responsable. & 1.22 & 1.13 \\
\hline 10. Fomentar el diálogo. & 1.37 & 1.16 \\
\hline 11. Ser audaz. & 1.36 & 1.4 \\
\hline \multicolumn{2}{|c|}{ Nota: mín 1, max 3} \\
\hline
\end{tabular}

Tabla 8 Comparativo de medias sobre los valores y cualidades que requiere el líder de hoy entre ambos estudios

$\mathrm{Al}$ analizar las medias de ambos estudios, se encontró que en el estudio de Omiziaki (2007), tener claros los objetivos que se quieren alcanzar con una media de 1.01 fue la de mayor importancia para los integrantes de la muestra, seguida de tener capacidad para enfrentar los retos (1.06), tener carácter (1.06) y ser honesto (1.07). En el caso del estudio aquí presentado la de mayor relevancia fue la de ser honesto (1.13), tener claro los objetivos que quiere alcanzar (1.14) y tener capacidad para enfrentar los retos (1.16). Como se puede observar se presentaron similitudes en los hallazgos respecto a los valores y cualidades que requiere el líder de hoy. Respecto a los dilemas y problemas éticos la tabla 9 muestra un comparativo entre ambos estudios.

\begin{tabular}{|l|r|r|}
\hline \multicolumn{1}{|c}{ Criterio de valoración } & Media & $\begin{array}{c}\text { Media } \\
\text { Omiaziki }\end{array}$ \\
\hline 1. Relaciones personales. & 1.51 & 1.73 \\
\hline 2. Liderazgo. & 1.51 & 1.50 \\
\hline 3. Religioso. & 1.78 & 1.80 \\
\hline 4. Familiar. & 1.65 & 1.39 \\
\hline 5. Cultura. & 1.77 & 1.34 \\
\hline 6. Educativo. & 1.40 & 1.24 \\
\hline 7. Político. & 1.56 & 1.16 \\
\hline 8. Económico. & 1.48 & 1.10 \\
\hline Nota: mín 1, max 3 & \\
\hline \multicolumn{2}{|l|}{} \\
\hline
\end{tabular}

Tabla 9 Comparativo de media entre ambos estudios en donde se encuentran los dilemas éticos

Según el estudio de Omiaziki (2007) los mayores dilemas éticos se presentan en el ámbito económico (1.10), seguido del político (1.16), y en el educativo con (1.24). En el caso del presente estudio en el ámbito educativo con una media de 1.40, seguido del económico (1.48), y con el mismo valor en sus medias en las relaciones personales y liderazgo con 1.51 respectivamente. Se ve una diferencia entre ambos estudios, coincidiendo en el ámbito educativo, aunque no con el mismo valor de las medias.

VELASCO-CEPEDA, Raquel Ivonne, CLARK-MENDÍVIL, Yesenia y ESPARZA-GARCÍA, Irma Guadalupe. Liderazgo actual: una percepción de estudiantes de educación superior. Revista Teoría Educativa. 2019 
Continuando con el análisis de los resultados encontrados en esta investigación, se puede retomar lo descrito por Perrone et al. (2013) en donde un determinante del comportamiento ético o no ético tiene que ver con la religiosidad. Esto se ratifica tanto en el estudio de Omiaziki (2007), y en la presente investigación en donde las medias más altas se obtuvieron en el ámbito religioso, significando con ello que efectivamente influyen en el tipo de comportamiento.

Terrazas (2015) hizo mención a que el ser humano en la sociedad del conocimiento se ha convertido en una persona que exige derechos, pero no desea asumir responsabilidades. A este respecto el estudio demostró que la percepción de los estudiantes estaba de acuerdo que cada vez hay más gente que no cumple con sus responsabilidades obteniendo una media de 4.18.

\section{Conclusiones y recomendaciones}

Entre las conclusiones se encuentran las siguientes:

(a) Se cumplió con el objetivo de la investigación que es identificar la percepción de los estudiantes respecto al liderazgo ante las problemáticas actuales, así como los valores y cualidades que requiere el líder de hoy ante los dilemas éticos.

(b) Respecto a los resultados del liderazgo ante las problemáticas actuales, se encontró que el que tuvo una media más alta fue el liderazgo actual necesita mucho de la conducta ética y moral, en segundo término se tiene el que la sociedad no ha tomado conciencia de sus grandes problemas, y tercero, las personas están decepcionadas por la falta de voluntad de las autoridades.

(c) Sobre la percepción de valores y cualidades que requiere el líder de hoy, se obtuvieron los siguientes hallazgos como los más relevantes según los integrantes de la muestra: ser honesto, tener claro los objetivos que quiere alcanzar, tener capacidad para enfrentar los retos, ser responsable y perseverante. (d) En relación con los ámbitos en donde se encuentran los dilemas éticos, el orden de importancia que prevaleció fue primeramente el educativo, seguido del económico, y en el liderazgo y las relaciones personales.

(e) Tratar de comprender el comportamiento del ser humano es complejo, aunado a las influencias que recibe del entorno, las circunstancias por las que atraviesa en un momento determinado, entre otros, pueden propiciar que se presenten dilemas éticos; $\mathrm{y}$ si no cuenta con valores y virtudes que le den guía a su toma de decisiones; puede desencadenar en comportamientos erróneos y poco éticos.

(f) El líder y los seguidores son elementos clave en las organizaciones; comúnmente se hace referencia a las características que debe poseer un líder; sin embargo, es importante conocer las cualidades de los seguidores. Castro, Clemenza \& Araujo (2012) mencionan que el proceso de liderazgo es dual: el líder influye sobre sus seguidores y también es influido por ellos.

(g) Los resultados obtenidos servirán como marco de referencia para futuras investigación, así como también contribuyen al estado del arte. continuación:

Las recomendaciones se presentan a

(a) Crear programas de difusión respecto a las problemáticas actuales, la importancia de los valores y cualidades del liderazgo antes los diferentes ámbitos de actuación.

(b) Vincular a la universidad con el sector productivo, social, religioso, familiar para trabajar en programas que contribuyan a la solución de las problemáticas encontradas en la presente investigación.

(c) Orientar acciones hacia la interior de la universidad, entre las que se podrían tener conferencias, revisar los programas de curso, foros de discusión, entre otros. 


\section{Referencias}

Álvarez del Blanco,R. (2008). Tú marca personal. $1^{\mathrm{a} e d i c i o ́ n . ~ E s p a n ̃ a: ~ E d i t o r i a l ~ P e a r s o n ~}$ Educación, S.A.

Castro, E.,Clemenza， C.,\&Araujo,R.(2012). Líderes y seguidores: una relación dual.Multiciencias, vol.12, pp.251-255

Contreras, F. (2008). Liderazgo: perspectivas de desarrollo e investigación. International Journal of Psychological Research, Vol.1, pp 64-72

Etling, A.(2005). Liderazgo efectivo. Técnicas, recursos y actividades didácticas en la dinámica de grupo. Sevilla: Trillas

Ferrer, J. y Clemenza,C. (2006). Dimensión ética del liderazgo como transparencia del poder local. Multiciencias, vol.6, núm. 1, pp.0-22

Franklin, E. y $\quad$ Krieger, M.(2011).Comportamiento organizacional. Enfoque para América Latina. México: Pearson Educación de México,S.A. de C.V.

George,D.y Mallery,P. (2003). SPSS for Windows step by step: A Simple Guide and Reference.11.0 Update.4aed. Boston: Allyn\&Bacon.

González González, Odris, \& González González, Oramis. (2008). Percepciones de los estudiantes universitarios, frente al liderazgo del docente. Revista Mexicana de Orientación Educativa, 5(13), 37-43

Gómez Ortíz, A. (2008). El liderazgo empresarial para la innovación tecnológica en las micro, pequeñas y medianas empresas. Revista Pensamiento y Gestión. No.24, pp157194

Griffin, R. (2011). Administración. 10a.ed. México: Cengage Learning Editores,S.A. de C.V

Hellriegel, S. y Slocum, J. (2009). Comportamiento organizacional. $12^{a}$ edición.México: Cengage Learning Editores, S.A. de C.V.

Kotter, J.P (1990). A force for change. New York, USA: The Free Press.
Lamb, C.,Hair,J.\& McDaniel,C. (2011). Marketing. $11^{\mathrm{a} e d i c i o ́ n . ~ M e ́ x i c o: ~ C e n g a g e ~}$ Learning Editores.

Lussier,R. y Achua,Ch.(2011). Liderazgo. Teoría, aplicación y desarrollo de habilidades. $4^{a}$ edición.México: Cengage Learning Editores, S.A. de C.V.

Méndez, C.(1995).Metodología. Guía para elaborar diseños de investigación en ciencias económicas, contables $\quad y$

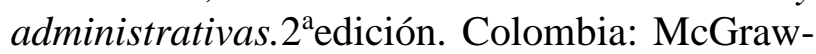
Hill/Interamericana,S.A.

McDaniel,C. y Gates,R. (2011). Investigación de

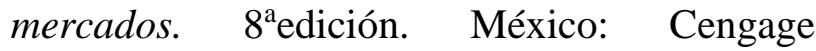
Learning Editores,S.A.

Münch, L. (2010). Administración. Gestión organizacional, enfoques y proceso administrativo. México: Pearson Educación de México, S.A.

Nelson, D. y Quick, J. (2013). CORG. Comportamiento

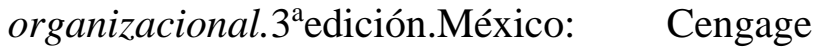
Learning Editores, S.A. de C.V,

Omiaziki,J.M. (2007). La percepción de los estudiantes universitarios acerca de las características más importantes que debe cubrir un líder para enfrentar los grandes problemas contemporáneos. Investigación universitaria multidisciplinaria. Año 6, No.6, pp.55-66

Perrone,

C.

M.,Engelman,S.,Filho,R.,\&Reinaldo,A.(2013).

Desafíos contemporáneos de la ética y de la moral en las organizaciones. Invenio,vol.17, num.31-32, pp.33-47

Prieto Pulido,R.A. y Fabelo,R.A.(2009). Liderazgo y capital social: Uso de redes como herramienta para el desarrollo sostenible. Telos, vol, núm.1, pp.52-68 
Ruíz-Cano, J., Cantú-Quintanilla, G. R., ÁvilaMontiel, D., Gamboa-Marrufo, J. D., JuárezVillegas, L. E., de Hoyos-Bermea, ., ChávezLópez, A., Estrada-Ramírez, K. P., MereloArias, C.A., Altamirano-Bustamante, M. M., de la Vega-Morell, N., Peláez-Ballestas, I., Guadarrama-Orozco, J. H., Muñoz-Hernández, O., Garduño-Espinosa, J., \& Grupo de Estudio sobre Dilemas Éticos HIMFG-IPADE, (2015). Revisión de modelos para el análisis de dilemas éticos. Boletín médico del Hospital Infantil de México, 72(2), 89-98.

Ruiz-Palomino, P., Ruiz-Amaya,C. y MartínezCañas,R. (2012). Cultura organizacional ética y generación de valor sostenible. Investigaciones Europeas de Dirección y Economía de la empresa, vol.18, núm 1,pp.17-31

Terrazas Pastor, R. (2015). Liderazgo tridimensional. PERSPECTIVAS, núm.35, pp.7-28

Vázquez Toledo,S., Bernal Agudo,J.L.,\& Liesa Orús,M. (2014). La conceptualización del liderazgo: una aproximación de la práctica educativa. REICE, Revista Iberoamericana sobre Calidad, Eficacia y Cambio en Educación, vol.12 num.5, pp.79-97

Williams, Ch. (2013). ADMON.

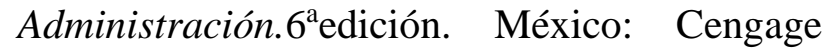
Learning Editores, S.A. de C.V 